\title{
Richard T. Gray, About Face. German Physiognomic Thought from Lavater to Auschwitz. 2004
}

\section{Journal Article}

Author(s):

Gamper, Michael

Publication date:

2006

Permanent link:

https://doi.org/10.3929/ethz-b-000422498

Rights / license:

In Copyright - Non-Commercial Use Permitted

Originally published in:

Arbitrium 24(2), https://doi.org/10.1515/ARBI.2006.204 
Richard T. Gray, About Face. German Physiognomic Thought from Lavater to Auschwitz. Wayne State University Press, Detroit 2004. LVI/453 S., € 4I,90.

Die Geschichte der Physiognomik und ihrer vielfältigen und weitreichenden Einflüsse beziehungsweise Reflexe in der Kultur der Moderne seit dem ausgehenden I8. Jahrhundert haben sich in den letzten is Jahren eines regen For- 
schungsinteresses erfreut. Vor allem kulturanthropologische, aber auch wissenschaftsgeschichtliche Studien haben physiognomische Praxis und Denken als mit grundlegenden Strukturen westlicher Kultur verbunden erwiesen. Dies gilt sowohl für Sammelbände, die eher literaturwissenschaftlich ausgerichtet sind, ${ }^{\mathrm{I}}$ als auch für solche, die dezidiert einen allgemeineren kulturwissenschaftlichen Fokus aufweisen ${ }^{2}$ beziehungsweise sich den Verflechtungen mit den Wissenschaften verschrieben haben. ${ }^{3}$ In dieses detailliert und gut erschlossene Forschungsfeld hat sich Richard T. Gray mit seiner umfangreichen Studie begeben, welche die Reihe der prominenten Arbeiten aus dem angloamerikanischen Raum zur Problematik der Physiognomik fortsetzt. ${ }^{4}$ Gray umreißt dabei mit seiner Monographie zeitlich fast vollständig den Bereich der modernen Physiognomik, indem er ihren Weg im „German Physiognomic Thought from Lavater to Auschwitz" beschreibt. Ihn leitet das spezifische Interesse an der Frage, „why physiognomic theory enjoyed such uncommon vitality in the sociocultural and intellectual atmosphere of modern Germany in particular" (S. XIX). Dabei erkennt er die strategische Funktion der Physiognomik in ihrer Eignung als angewandte Hermeneutik, also als spezifische interpretative Praxis im Dienste von Nationalismus und Rassismus (S. XXII).

Dieser bestimmte Fokus ist in der Lage, das reichhaltige Material auf diese Thematik hin zu selektieren und zu organisieren, hat aber auch zur Folge, daß sich Gray erstens fast ausschließlich auf deutsche Zeugnisse stützt, zweitens stark zu nationenspezifischen ideengeschichtlichen Konzeptualisierungen neigt und drittens seiner Studie, trotz gegenteiliger Beteuerung, eine teleologische Tendenz unterlegt, 5 die oft allzu direkt vom Ausgangspunkt (Lavater) zum Ziel (nationalsozialistische Rassentheorie) führt - der Kategorienfehler im Titel („from Lavater to Auschwitz“) verrät diese problematische Tendenz der Arbeit bereits. Hier fordern die Thesenstärke des Buchs und dessen ideologiekritischer und ideengeschichtlicher Zugriff ihren Tribut in der Form von Verknappungen und verzeichnenden Zuspitzungen, die mit einer wissensgeschichtlich-diskursanalytischen methodischen Orientierung wohl zu vermeiden gewesen wären. Unterteilt ist der Hauptteil der Arbeit in sieben Kapitel, die gerahmt werden von einer ausführlichen Einleitung

\footnotetext{
${ }^{\mathrm{I}}$ Wolfram Groddeck / Ulrich Stadler (Hgg.), Physiognomie und Pathognomie. Zur literarischen Darstellung von Individualität. Berlin 1994.

${ }^{2}$ Claudia Schmölders (Hg.), Der exzentrische Blick. Gespräch über Physiognomik. Berlin 1996; Claudia Schmölders / Sander L. Gilman (Hgg.), Gesichter der Weimarer Republik. Eine physiognomische Kulturgeschichte. Köln 2000; Karl Pestalozzi / Ulrich Stadler (Hgg.), Im Lichte Lavaters. Zürich 2003.

3 Rüdiger Campe / Manfred Schneider (Hgg.), Geschichten der Physiognomik. Text, Bild, Wissen. Freiburg/Br. I996.

${ }^{4}$ Als jüngstes Beispiel vgl. Melissa Percival / Graeme Tytler (Hgg.), Physiognomy in Profile. Lavater's Impact on European Culture. Newark 2005.

5 "Moreover, this nonlinear methodological procedure is intended to discourage any implication that eighteenth-century physiognomic thought and twentieth-century racism are linked by a relationship of teleological necessity“ (S. XXVI). Dem widerspricht aber die Praxis, daß in den einzelnen Kapiteln zu bestimmten historischen Positionen der Physiognomik die Gemeinsamkeiten mit späteren Exponenten, vor allem völkischen und nationalsozialistischen Autoren, hervorgehoben werden.
} 
und einer „Conclusion“, die sich den bildlichen Reproduktionstechniken zuwendet, die das jeweilige visuelle historische Apriori der Physiognomik bilden - eine essentielle Perspektive, deren stärkere Berücksichtigung in den einzelnen Abschnitten der Studie durchaus wünschenswert gewesen wäre.

In Kapitel I wendet sich Gray den semiotischen Grundlagen der Lavaterschen Physiognomik zu und sieht diese untrennbar verbunden mit den Aporien einer aufklärerischen Zeichentheorie, welche die Zeichen als transparente Bedeutungsträger definiert und ihnen keine Eigenwertigkeit zugestanden habe. Lavaters Absicht, alle kontingenten Aspekte aus seiner physiognomischen Analyse zu tilgen und sich möglichst auf die unveränderlichen Teile des Gesichts zu konzentrieren, entspreche die Ausschließlichkeit, mit der er alle zeichenpraktische Arbitrarität zu vermeiden versuche. Dabei habe er die Schwächen dieser Semiotik kaschiert mit einer pathetischen Formulierungsweise, die er dem Sturm und Drang verdanke. Darin sieht Gray eine "marriage of logic and rhetoric" (S. 55) gestiftet, die charakteristisch bleibe für die weiteren modernen deutschen Physiognomik-Theorien.

Die zeichentheoretische Perspektive wird im zweiten Kapitel weiterverfolgt, wo es um die semiotische Konstitution moderner Individualitäten geht. Dort werden Lavaters Physiognomik und Franz Joseph Galls Phrenologie einer Position zugeschlagen, die Individualität versteht als ein selbstidentisches inneres Sein, das sein unveränderliches Wesen mimetisch in den sinnlich wahrnehmbaren Teilen des Körpers reproduziert. Die Opponenten gegen diese Haltung, unter denen sich Gray vor allem auf Georg Christoph Lichtenberg als Gewährsmann stützt, konzeptualisierten dagegen den Menschen als ein autonomes, sich selbst hervorbringendes und so in ständigem Transformationsprozeß stehendes Individuum. Dabei erweise sich die physiognomische Position über die Jahrhunderte als resistent gegen die Argumente dieser Gegenposition, und es gelinge ihr im Gegenteil, die Kritik der Gegner, wie etwa diejenige Hegels, ins eigene System zu inkorporieren.

In Kapitel 3 verfolgt der Verfasser, auf welche Weise die humanistischen Tendenzen in Lavaters Werk und in den Schriften von Carl Gustav Carus unterminiert werden von materialistischen Überzeugungen und einem empirizistischen Primat, die rassistischen Vorurteilen Tür und Tor öffnen. Dabei kann sich Gray vor allem auf den vierten Band von Lavaters Physiognomischen Fragmenten und Carus' Ueber ungleiche Befähigung der verschiedenen Menschbeitsstämme für höhere geistige Entwicklung von I 849 stützen. Getragen würden diese Tendenzen von Carus' „radically positivistic metaphysics“ (S. I I8), die in Lavater einen Vorläufer hätten, der den „turn toward codifying physiognomics as a strictly positivistic and empirical science" ausgelöst habe (S. XVIIIf.).

Weiter wird dann Goethe in einer doppelten Funktion porträtiert: Im vierten Kapitel erscheint er einerseits als Begründer einer Morphologie, die als dynamisches Konzept der Erfassung des Innen-Außen-Bezugs von Lebewesen eine Alternative zur statischen Physiognomik Lavaterscher Prägung darstellt, und andererseits als eine Autorität, die von späteren Verfassern als Gründungsvater für die verschiedensten Anliegen in Anspruch genommen worden ist. Dies beginne mit Carus' oben genannter Schrift, die als Denkschrift zum bundertjäbrigen Geburtsfeste Goethe's erschienen ist, und zieht 
sich später von Ludwig Klages’ antifreudianischer „Seelenkunde“ über Oswald Spenglers intuitive Lebensphilosophie bis hin zu Rudolf Kassners „universaler Physiognomik“.

Das fünfte Kapitel wendet sich dem von ungemein vielen Exponenten getragenen Revival der Physiognomik in der Weimarer Republik zu, die Gray sozialgeschichtlich ähnlich wie die Hausse im I 8. Jahrhundert mit einem gravierenden sozialen Umbruch erklärt, der neue Weisen der visuellen Orientierung in einem undurchschaubar gewordenen Alltag erfordert habe. Im Zentrum des Booms der 1920er und I930er Jahre stehe nun die Favorisierung von intuitiven vor rationalen Formen des Denkens, die sich im Zeichen von Lebensphilosophie, Goethe-Verehrung und Antimodernismus beziehungsweise Kulturpessimismus figuriere (S. I79f.). Auch hier sind es Spengler und Kassner, auf deren Werke sich die Analyse richtet; deren Ziel ist es, den Anteil der beiden Autoren am völkischen Denken zu bestimmen.

Damit sind die Kapitel 6 und 7 vorbereitet, die sich nun den dezidiert völkisch ausgerichteten physiognomischen Theorien von Hans F. K. Günther und Ludwig Ferdinand Clauss zuwenden, denen auch im Nationalsozialismus große Bedeutung zukam. Gray konzentriert sich bei der Analyse dieser Theorien auf deren Genealogie, also auf ihre spezifische Rezeption von Elementen aus der deutschen Ideengeschichte. Dabei seien es im Fall von Günther vor allem die materialistische Linie der Physiognomik und die Rassetheorien von Gobineau und Chamberlain, die zu einer einflußreichen und weitverbreiteten Ideologie verbunden würden. Hier besteht das Verdienst von Gray darin, darüber hinaus auch weitere Traditionsfiliationen aufzuzeigen, die in Günthers Werk eine wichtige Rolle spielen; es sind vor allem verschiedene Ausformungen der Lebensphilosophie, die als Nährboden der Güntherschen Physiognomik namhaft gemacht werden können. Im Falle von Clauss' - im Gegensatz zu Günthers ethnologisch ausgerichteter Physiognomik eher psychologisch orientierter - Lehre geht Gray dann vor allem der Husserl-Rezeption nach, wobei es ihm gelingt, auch die Subtilität der Argumentationsweise von Clauss herauszuarbeiten. Rassistische Physiognomik zur Zeit des Nationalsozialismus, und dies ist das Hauptanliegen und -ergebnis dieser Kapitel und auch des ganzen Buches, ist deshalb nicht die Folge eines Mißbrauchs einer ehrwürdigen Tradition. Vielmehr zeigt Gray Günther und Clauss als „direct heirs to central theoretical tenets and methodological practices generated by German physiognomists since Lavater" (S. 33I).

Grays Buch bietet so eine prägnante und materialreiche Darstellung des physiognomischen Denkens in Deutschland in seinen verschiedenen Facetten. Er zeigt, wie sich eine ,materialistisch-wissenschaftliche' und eine, intuitive' Linie mit Lavater und Goethe als durchaus retrospektiv konstruierten und phantasmatischen Gründungsvätern herauskristallisierte, die sich dann, vor allem in der Weimarer Republik, in vielen historischen Konstellationen überlagerten. Dadurch gelingt Gray hinsichtlich der geistesgeschichtlichen Filiationen eine komplexe Darstellung der Thematik, die auch in den völkischen Entwürfen ein differenziertes Konglomerat von Traditionen erkennt. Er hat damit eine in den Grundthesen schon länger etablierte physiognomikkritische Perspektive material und argumentativ stark unterfüttert und ein Grundlagenwerk dieser Forschungsrichtung geschrieben. 
Prekär an Grays groß angelegter Konstruktion einer spezifisch ,deutschen intellektuellen Tradition sind dabei aber vor allem drei Dinge. So benutzt er erstens sehr allgemeine, nicht genauer definierte Begriffe von „materialistic“, „positivistic“ und „scientific“, um die von Lavater ausgehende, latent rassistische Tradition zu kennzeichnen. Damit aber ignoriert er, zweitens, den grundlegenden epistemologischen Bruch, der sich durch die Etablierung der empirischen Wissenschaften um die Mitte des 19. Jahrhunderts ereignet hat. Denn die Durchsetzung der wissenschaftlichen Praktiken des Messens, des Zählens und der statistischen Auswertung hat den ,wissenschaftlichen Materialismus' auf eine neue Basis gestellt, weshalb die Differenzen zu den vornehmlich in Frankreich verbreiteten physiologischen Materialismen des I 8. Jahrhunderts weit größer sind als die Gemeinsamkeiten. Signifikanterweise berücksichtigt Gray weder die Anthropometrie noch die Kriminalanthropologie des späten 19. Jahrhunderts und deren Kategorisierungsschemata der Innen-Außen-Relation; hätte er dies getan, hätte sich freilich herausgestellt, daß die vermeintlich genuin deutsche physiognomische Tradition des beginnenden 20. Jahrhunderts wesentlich auf Prämissen aufruht, die nur als Aspekte eines gesamteuropäischen Unternehmens zu verstehen sind. Da Gray aber diese wichtigen Umbrüche im Denken über den Körper und dessen Signifikanz übergeht, weist sein Buch eine weiter nicht thematisierte historische Lücke in der zweiten Hälfte des 19. Jahrhunderts auf, was wiederholt Engführungen zur Folge hat, die Konzepte des I8. Jahrunderts und Entwürfe aus der Zeit der Weimarer Republik allzu unmittelbar in Beziehung setzen. Grays Methode verläßt sich deshalb, drittens, zu oft auf den strukturellen Vergleich, der zur Gewinnung von gemeinsamen Momenten die Unterschiede zu sehr zurückstellt. Besonders deutlich wird dies in der „Introduction“, in der über einen "fundamental distrust of human intentionality" ein „single intellectual-historical continuum “ zwischen Lavaters „physiognomic surface hermeneutics“ und Freuds „psychoanalytical depth hermeneutics" postuliert (S. XLIX) beziehungsweise in der Seele-Körper-Relation von Lavaters Physiognomik und Husserls Phänomenologie ein wesentlicher $\mathrm{Zu}$ sammenhang gesehen wird (S. LIII). Hier wird, wie oft in dieser Arbeit, auf - gerade hier: vage - strukturelle Analogien vertraut, die letztlich aber den Gegenstand eher verdunkeln als erhellen.

ETH Zürich

SNF-Förderungsprofessur für Literaturwissenschaft

Michael Gamper

Rämistrasse IOI

$\mathrm{CH}-8006$ Zürich

migamper@ethz.ch 